\title{
Role of Echocardiography In Acute Rheumatic Fever Compared With Jones Criteria
}

\author{
Sarkar M K ${ }^{1}$, Malviya $K^{2}$, Barpha A $S^{3}$, Lalchandani $A^{4}$, Dey $S^{5}$ \\ ${ }^{1}$ Dr Manuj Kumar Sarkar, Assistant Professor of Medicine, Index Medical College Hospital and Research Centre, Indore, \\ MP, India, ${ }^{2}$ Dr Kamal Malviya, Assistant Professor of Medicine, Index Medical College Hospital and Research Centre, \\ Indore, MP, India, ${ }^{3} \mathrm{Dr}$ Amit Singh Barpha, Assistant Professor of Medicine, Index Medical College Hospital and \\ Research Centre, Indore, MP, India, ${ }^{4}$ Dr Arati Lalchandani, professor of Medicine, GSVM Medical College, Kanpur, UP, \\ India, ${ }^{5}$ Dr Subhra Dey, Senior Lecturer, Index Institute of Dental Science, Indore, MP, India
}

Address for Correspondence: Dr Manuj Kumar Sarkar, Email: manojsarkar654321@gmail.com

\begin{abstract}
Introduction: Rheumatic heart disease (RHD), the sequel of acute rheumatic fever (ARF), is a very common cause of cardiovascular mortality and morbidity. RHD results in enormous disease burden translating into huge economic and social losses in India. Classical ARF is still encountered across the country. The diagnosis of RF remains clinical. Echocardiography is a very important diagnostic tool to detect valvular lesions in patients with carditis of acute rheumatic fever. Jones criteria has been modified several times but the role of echocardiography in detecting carditis is not been mentioned in any modifications. Methods and Material: The study was conducted from November 2007 to October 2009, for duration of 2 years in GSVM medical college, Kanpur. Patients with joint pain (arthritis, arthralgia), chest pain, dyspnoea, palpitation, chorea (involuntary movements), subcutaneous nodule, erhythema marginatum, sore throat, skin infection, recurrent upper respiratory tract infection etc, who visited OPD, health camps, or were admitted in Medicine and Pediatrics department were included in the study. Thus selected patients were undergone detailed history taking, clinical examinations particularly they were evaluated to find out whether they were having symptom/signs of carditis and fulfilling Jones criteria to diagnose Acute Rheumatic fever. The same patients were evaluated with Echocardiography to detect carditis. Results: 40 patients were diagnosed as acute rheumatic fever based on revised Jones criteria. Only 24 patients showed carditis clinically, but Echocardiography showed 27 patients with evidence of carditis. Ventricular septal defect was also detected in 3 patients, which was wrongly detected as carditis in clinical examination. 21 patients had carditis clinically, but echocardiography diagnosed carditis in 27 patients. (p value $<0.05$ ). Conclusion: Echocardiography plays a very important role in detection of carditis. Echocardiographic confirmation of carditis could be accepted as major criteria.
\end{abstract}

Keywords: Acute Rheumatic Fever, Carditis, Echocardiography, Jones Criteria

\section{Introduction}

Rheumatic heart disease (RHD), the sequel of acute rheumatic fever (ARF), is a very common cause of cardiovascular mortality and morbidity [1]. It is estimated that a minimum of 15.6 million people have RHD in the world, with 2, 82,000 new cases being added each year, and is estimated to cause 2, 33,000 deaths each year [2]. RHD results in enormous disease burden translating into huge economic and social losses in India. Classical ARF is still encountered across the country. The diagnosis of RF remains clinical [3]. The

Manuscript received: $30^{\text {th }}$ Apr 2015

Reviewed: $14^{\text {th }}$ May 2015

Author Corrected: $27^{\text {th }}$ May 2015

Accepted for Publication: $13^{\text {th }}$ June 2015 original Jones Criteria as proposed by Dr. T. Duckett Jones have been modified several times and according to updated revised criteria major manifestations are carditis, polyarthritis, chorea, erythema marginatum and subcutaneous nodules. Minor manifestations include fever, arthralgia and laboratory findings of elevated erythrocyte sedimentation rate, C-reactive protein and prolonged PR interval on ECG. For making a diagnosis of acute rheumatic fever, two major, or one major and two minor manifestations must be accompanied by supporting evidence of antecedent group A streptococcal infection in the form of positive throat culture or elevated or rising anti-streptolysin titre. Role 
of echocardiography has not been defined in these modifications but may be important, as clinical detection of soft murmurs may be difficult [4].

A significant number of patients with suspected acute rheumatic carditis have no clinical murmurs but have documented regurgitation on echocardiography. Subclinical carditis is relatively common in RF. Thus, a new category of subclinical carditis has emerged [3]. Subclinical carditis is relatively common in RF. A metaanalysis of various studies on subclinical carditis reported to $18.1 \%$ (95\% CI 11.1-25.2) [5]. In areas with a high prevalence of $\mathrm{RF}$, the consequences of underdiagnosis are likely to be greater than overdiagnosis. Recent analyses have suggested that subclinical carditis as a major Jones criterion influences the diagnosis of acute RF in $16 \%$ of patients [6].

Although RF is a systemic disease with multiorgan involvement, none of its manifestations, except for carditis, lead to permanent damage. Detection of active rheumatic carditis is of great prognostic and therapeutic importance and is currently based on the Jones criteria. Not infrequently, the diagnosis of carditis by the Jones criteria becomes difficult, especially when carditis is the isolated manifestation of the disease or when the rheumatic activity occurs on preexisting RHD [7, 8, 9, and 10]. It is important to develop a diagnostic strategy that will improve our ability to diagnose rheumatic carditis and allow us to apply existing criteria more efficiently [7, 10, and 11]. The advent of modern, highly sensitive cardiac imaging modalities, predominantly echocardiography/Doppler ultrasound (echo-Doppler), has raised the question whether the
Jones criteria should be modified to incorporate these techniques [7]. Echocardiography along with Doppler assessment gives excellent details of the structural and functional abnormalities in acute RF. Yet, echocardiography is not included as a criterion in the diagnosis of RF [3].

The study was done in a tertiary care centre to see the role of echocardiography in diagnosing acute rheumatic fever compared with Jones criteria.

\section{Methods and Material}

The study was conducted from November 2007 to October 2009, for duration of 2 years in GSVM medical college, Kanpur. Non probability sampling method with an intension for purposive sampling was used to collect the subjects. Inclusion Criteria: Patients with joint pain (arthritis, arthralgia), chest pain, dyspnoea, palpitation, chorea (involuntary movements), subcutaneous nodule, erhythema marginatum, sore throat, skin infection, recurrent upper respiratory tract infection etc, who visited OPD, health camps, or were admitted in Medicine and Pediatrics department were included in the study. Exclusion Criteria: Patients not fulfilling above criteria were excluded.

Thus selected patients were undergone detailed history taking, clinical examinations particularly they were evaluated to find out whether they were having symptom/signs of carditis and fulfilling Jones criteria to diagnose Acute Rheumatic fever. The same patients were evaluated with Echocardiography to detect carditis.

\section{Results and Statistical Analysis}

Using modified Jones criteria, 40 patients were diagnosed as Acute Rheumatic fever. 14 patients were male and 26 patients were female. Most common age group were 10-15 years, $45 \%$ patients were from this group.

Table I: Age distribution of Acute Rheumatic fever

\begin{tabular}{|l|l|l|}
\hline Age & Number of Patients & Percentage of Patients \\
\hline $5-10$ years & 7 & $17.5 \%$ \\
\hline $10-15$ years & 18 & $45 \%$ \\
\hline $15-20$ years & 9 & $22.5 \%$ \\
\hline $20-25$ years & 6 & $15 \%$ \\
\hline
\end{tabular}


Table II: Sex distribution of Acute Rheumatic fever

\begin{tabular}{|l|l|l|}
\hline Gender & Number of Patients & Number of patients \\
\hline Male & 14 & $35 \%$ \\
\hline Female & 26 & $65 \%$ \\
\hline
\end{tabular}

Table III: Distribution of major criteria in patients of Acute Rheumatic fever:

\begin{tabular}{|l|l|l|}
\hline & Nuber of Patients & Percentage \\
\hline Polyarthritis & 33 & $82.5 \%$ \\
\hline Carditis( clinically) & 24 & $60 \%$ \\
\hline Chorea & 2 & $5 \%$ \\
\hline Subcutaneous nodule & 1 & $2.5 \%$ \\
\hline Erythema Marginatum & 0 & 0 \\
\hline
\end{tabular}

Table IV: Distribution of minor criteria in patients with ARF:

\begin{tabular}{|l|l|l|}
\hline Minor Menifestation & Number of Patients & Percentage \\
\hline ASO titre raised & 36 & $90 \%$ \\
\hline Arthralgia & 36 & $90 \%$ \\
\hline Fever & 25 & $62.5 \%$ \\
\hline PR Prolongation & 7 & $17.5 \%$ \\
\hline Throat culture & 5 & $12.5 \%$ \\
\hline
\end{tabular}

Table V: Echocardiograhic detection of cardiac lesions in patents of ARF:

\begin{tabular}{|l|l|l|l|}
\hline Echocardiographic finding & Number of Patients & Percentage \\
\hline \multirow{3}{*}{ Carditis } & Mitral regurgitation & 20 & $50 \%$ \\
\cline { 2 - 4 } & Aortic regurgitation & 3 & $7.5 \%$ \\
\cline { 2 - 4 } & Pericardial effusion & 4 & $10 \%$ \\
\hline \multicolumn{2}{|l|}{ Congenital Heart Disease( VSD ) } & 3 & $7.5 \%$ \\
\hline
\end{tabular}

Among 40 patients diagnosed as ARF using Jones criteria, among major criterias polyarthritis ( $\mathrm{n}=33$ ) and overall arthralgia and raised ASO titre $(n=36)$ was the most common presentation.

Only 24 patients had carditis clinically, but using Echocardiography, 20 patients showed mitral regurgitation (MR) suggestive of rheumatic mitral valvulitis, 3 patients showed aortic regurgitation (AR), suggestive of aortic valvulitis, 4 patients showed pericardial effusion suggestive of rheumatic involvement of pericardium. 27 patients showed evidence of carditis. Small ventricular septal defect was detected in 3 patients, which was wrongly diagnosed as carditis in clinical examination. So using Fisher's exact test $\mathrm{P}$ value $=0.0229$ was found, which can be considered to be statistically significant.

\section{Discussion}

Even in the golden era of clinical auscultation, a number of patients with no audible murmurs in the first attack of RF developed RHD on follow-up, suggesting that carditis was missed by clinical examination $[3,12]$

Murmur may be missed by experienced clinicians because of presence of tachycardia [3, 4].

Pre-existing valvular lesions can worsen due to recurrences; hence, accuracy in the diagnosis is very essential. Echocardiography not only confirms and quantifies valvular regurgitation but is also useful in establishing an alternative cause of murmur [3]. EchoDoppler examination identifies valvular regurgitation not detectable with clinical examination, [13] allows visualization of valve structure, and allows detection of unrelated causes of valve dysfunction, such as mitral valve prolapse.

In our study, out of the 40 patients diagnosed as acute rheumatic fever, polyarthritis was present in $33(82.5 \%)$ 
patients; Carditis was present in $24(60 \%)$ patients on clinical examination, though echocardiography showed carditis in $27(67.5 \%)$ patients. More importantly clinical examination wrongly detected carditis in 3 patients, who were suffering from Ventricular septal defect. So there was significant difference in detection of carditis by clinical examination and two dimensional echocardiography. Thus, Echocardiography is more sensitive than clinical assessment in detection of carditis in acute rheumatic fever $[4,13]$.

Moreover, Echocardiography also detected small Ventricular Septal defect in 3 patients, their murmurs were wrongly considered as carditis by clinical examination. Echocardiography not only confirms the diagnosis of carditis but also establishes an alternative cause of murmur which may be over diagnosed as rheumatic carditis. Echocardiography can avoid both over diagnosis and under diagnosis of carditis [4, 14].

Echocardiography is very useful for confirmation of carditis in those patients where clinical examination shows signs of pan-carditis. For example, signs of endocarditis like detection of new murmur or change in preexisting murmur, signs of myocarditis like signs of unexplained heart failure, signs of pericarditis like pericardial effusion or pericardial rub. But detection of all these by clinical examination is very difficult as murmurs appear even in the late phase of the disease when destruction of heart valves are overt, heart failure can be because of any cause other then myocarditis, pericardial friction rub is difficult to auscultate and even if it appears for very short period of time. So it is very difficult to diagnose carditis by clinical examination. Echocardiography can diagnose pancarditis easily. So using echocardiography to detect carditis will lead to early diagnosis of carditis [15].

The recent Australian [16] and New Zealand [17] guidelines on RF have accepted echocardiographic subclinical carditis as a major criterion. All patients with suspected or definite RF should undergo echocardiography to identify evidence of carditis. Such an approach is likely to avoid wrong diagnosis and minimize under diagnosis [3].

Hence, in India also we need to incorporate subclinical carditis as a major criterion as any other region with high prevalence of RF/RHD [3].

\section{Conclusion}

Females are more commonly affected in ARF. Joint involvement is most common presentation. Carditis is second commonest major manifestation. Chorea, subcutaneous nodule, erythema marginatum are rarely found.

Echocardiography plays a very important role in detection of carditis in the subclinical stage of the disease state, where we are not able to detect carditis by clinical examination. It is also important in confirmation of carditis when it is not detected by clinical examination. Echocardiography can also role out other aetiologies of cardiac murmur which can be miss diagnosed as rheumatic carditis.

Echocardiography is a very important toll for diagnosis of carditis in acute rheumatic fever. So echocardiography should be accepted as major criteria for diagnosis of acute rheumatic fever [10, 12].

Funding: Nil

Conflict of interest: Nil

Permission from Institutional Research Board (IRB): Yes

\section{References}

1. WHO Study Group. Rheumatic Fever and Rheumatic Heart Disease. WHO Technical Report Series No. 764. Geneva: World Health Organization; 1988.

2. WHO. Rheumatic fever and rheumatic heart disease: Report of a WHO Expert Consultation. WHO Technical Report Series. vol. 923. Geneva: World Health Organization; 2004. Expert Consultation on Rheumatic Fever and Rheumatic Heart Disease (2001: Geneva, Switzerland).

3. Ramakrishnan S. Echocardiography in acute rheumatic fever. Ann Pediatr Cardiol. 2009 Jan;2(1):614. doi: 10.4103/0974-2069.52812.

4. Saxena A. Diagnosis of rheumatic fever: current status of Jones Criteria and role of echocardiography. Indian J Pediatr. 2000 Mar;67(3 Suppl):S11-4.

5. Tubridy-Clark M, Carapetis JR. Subclinical carditis in rheumatic fever: A systematic review. Int $\mathbf{J}$ Cardiol. 2007 Jun 25;119(1):54-8. Epub 2006 Oct 10.

6. Vijayalakshmi IB, Vishnuprabhu RO, Chitra N, Rajasri R, Anuradha TV. The efficacy of echocardiographic criterions for the diagnosis of 
carditis in acute rheumatic fever. Cardiol Young. 2008 Dec;18(6):586-92. doi: 10.1017/S1047951108003107. Epub 2008 Oct 10.

7. Narula J, Chandrasekhar Y, Rahimtoola S. Diagnosis of active rheumatic carditis. The echoes of change. Circulation. 1999 Oct 5;100(14):1576-81.

8. Markowitz M. Evolution and critique of changes in the Jones criteria for the diagnosis of rheumatic fever. $\mathrm{N}$ Z Med J. 1988 Jun 8;101(847 Pt 2):392-4.

9. Narula J, Reddy KS, Khaw BA. Can antimyosin scintigraphy supplement the Jones Criteria in the diagnosis of active rheumatic carditis? In: Khaw BA, Narula J, Strauss HW, eds. Monoclonal Antibodies in Cardiovascular Diseases. Philadelphia, Pa: Lea \& Febiger; 1994.

10. Narula J, Chopra P, Talwar KK, Vasan RS, Reddy KS, Tandon R, Bhatia ML, Southern JF. Endomyocardial biopsies in acute rheumatic fever. Circulation. 1993 Nov;88(5 Pt 1):2198-205.

11. Bhatnagar A, Calegaro JUM, Narula J. Radionuclide imaging in acute rheumatic fever. In: Narula J, Virmani R, Reddy KS, Tandon R, eds. Rheumatic Fever. Washington, DC: American Registry of Pathology Publication; 1999:329-338.
12. Feinstein AR, Stern EK, Spagnuolo M. The prognosis of acute rheumatic fever. Am Heart J. 1964 Dec;68:817-34.

13. Abernathy M, Bass N, Sharpe N, Grant C, Neutze J, Clarkson P, Greaves S, Lennon D, Snow S, Whally G. Doppler echocardiography and the early diagnosis of carditis in acute rheumatic fever. Aust N Z J Med. 1994 Oct;24(5):530-5.

14. Vijayalakshmi IB, Mithravinda J, Deva AN. The role of echocardiography in diagnosing carditis in the setting of acute rheumatic fever. Cardiol Young. 2005 Dec;15(6):583-8.

15. IB Vijayalakshmi. Rheumatic Fever, Rheumatic Heart Disease Registry and Control Program. IB Vijayalakshmi ed. Acute Rheumatic Fever and Chronic Rheumatic Heart disease. 2011. Jaypee Brothers Medical Publishers. India. P324-336.

16. Carapetis JR, Brown A, Wilson NJ, Edwards KN. Rheumatic Fever Guidelines Writing Group. An Australian guideline for rheumatic fever and rheumatic heart disease: An abridged outline. Med J Aust. 2007 Jun 4;186(11):581-6.

17. Atatoa-Carr P, Lennon D, Wilson N. New Zealand Rheumatic Fever Guidelines Writing Group. Rheumatic fever diagnosis, management, and secondary prevention: A New Zealand guideline. N Z Med J. 2008 Apr 4;121(1271):59-69.

\section{How to cite this article?}

Sarkar M K, Malviya K, Barpha A S, Lalchandani A, Dey S. Role of Echocardiography In Acute Rheumatic Fever Compared With Jones Criteria. Int J Med Res Rev 2015;3(5):502-506. doi: 10.17511/ijmrr.2015.15.097. 